\title{
Orientação acadêmica: percepções de discentes e docentes sobre a prática pedagógica na formação em Odontologia
}

\author{
Raul Elton Araújo Borges*, Bruno Cesar de Vasconcelos Gurgel**, Maria Cristina dos Santos \\ Medeiros**, Patrícia dos Santos Calderon** \\ * Doutorando, Programa de Pós-graduação em Ciências \\ Odontológicas, Universidade Federal do Rio Grande do Norte \\ ** Professor(a) Adjunto(a), Departamento de Odontologia, \\ Universidade Federal do Rio Grande do Norte
}

Recebido em 17/07/2019. Aprovado em 25/04/2020.

\begin{abstract}
RESUMO
O estudo objetivou avaliar o papel da orientação acadêmica e os desafios atuais relacionados a essa prática pedagógica durante a formação acadêmica em Odontologia, na percepção de docentes e discentes. Trata-se de estudo de natureza quantiqualitativa, desenvolvido no curso de Odontologia da Universidade Federal do Rio Grande do Norte (UFRN). A coleta de dados foi realizada por meio de questionários semiestruturados, respondidos por 106 estudantes e 15 professores de Odontologia com experiência em orientação acadêmica. A análise quantitativa dos resultados utilizou estatísticas descritivas e, na sequência, realizou-se avaliação qualitativa por meio da técnica de análise de conteúdo. As percepções discentes demostraram fragilidades no desempenho dos professores orientadores acadêmicos, principalmente em relação ao vínculo com a turma, período e frequência dos encontros, assim como metodologia adotada. Os docentes apontaram a necessidade de estratégias de educação permanente, visando sua qualificação para o exercício dessa função. Conclui-se que existe subaproveitamento dessa prática pedagógica na formação em Odontologia. Para superar esta fragilidade e os desafios inerentes à prática, na visão dos discentes é necessário que os orientadores acadêmicos se tornem mais presentes no cotidiano universitário desde o primeiro período, com orientações precoces sobre as suas obrigações e mediando o processo de formação acadêmica em todas as fases do curso. Para os docentes, é necessário investir em processos permanentes de desenvolvimento docente.
\end{abstract}

Descritores: Educação em Odontologia. Docentes de Odontologia. Desenvolvimento de Pessoal. 


\section{INTRODUÇÃO}

Em decorrência da implantação das Diretrizes Curriculares Nacionais (DCN) dos cursos de graduação em saúde em meados de 2002, muitas iniciativas com propostas de mudanças curriculares foram observadas em diversos cursos de Odontologia no país ${ }^{1,2}$. Tais mudanças foram pautadas principalmente na adoção de novas metodologias de ensino-aprendizagem, maior articulação entre as áreas de conhecimento, flexibilização curricular e busca de uma formação direcionada para as necessidades de saúde da população ${ }^{3}$. Sabe-se que as DCN não impõem um caráter único para os diversos cursos distribuídos no Brasil, mas definem uma base formadora sólida, sinalizando o que deve ser constantemente buscado pelas instituições acerca da qualidade do ensino ${ }^{4,5}$.

Contudo, o processo de mudança conferido pelas reformulações curriculares no ensino em Odontologia traz consigo inúmeros desafios, entre os quais romper com modelos de ensino tradicionais e formar profissionais de saúde com competências que lhes permitam recuperar a dimensão essencial do cuidado ${ }^{6,7}$. Vale destacar que essas discussões estão inseridas em um contexto mais amplo e são oriundas das conquistas obtidas ao longo dos últimos anos, tanto na área da Educação quanto na área da Saúde.

É nesse cenário que o curso de Odontologia da Universidade Federal do Rio Grande do Norte (UFRN) passou por reformulações e modificações profundas na sua matriz curricular a partir de 2015. Tal empreitada surgiu diante a necessidade de superar as fragilidades constatadas no currículo antigo vigente desde 2006, e das então novas exigências na formação de profissionais sinalizadas nas DCN para os cursos de Odontologia, que constituíram em última instância as bases norteadoras na elaboração de uma nova estrutura curricular e implementação de práticas pedagógicas inovadoras.

No entanto, este processo de transição e reestruturação curricular é lento e permeado de dificuldades, pois há a necessidade de uma complexa mudança estrutural, que implica na alteração de diversas práticas e cultura pedagógica das instituições de ensino superior (IES) ${ }^{8,9}$. Diante desse panorama, dentre as inúmeras mudanças que acompanham tal processo, o Núcleo Docente Estruturante (NDE) do curso de Odontologia da UFRN pautou como um desafio a ser discutido a estratégia da prática pedagógica utilizada pelos docentes orientadores acadêmicos na formação em Odontologia.

Em linhas gerais, na UFRN a orientação acadêmica tem como objetivo contribuir para a integração dos estudantes à vida universitária, orientando-os quanto às atividades acadêmicas, função regulamentada pela resolução no 171/2013CONSEPE/UFRN ${ }^{10}$, que discorre sobre as atribuições do orientador acadêmico. Dentre suas principais funções, destacam-se: colaborar com a coordenação e o NDE do curso na apresentação do projeto pedagógico e da estrutura universitária aos estudantes; acompanhar o desenvolvimento acadêmico dos estudantes sob sua orientação; planejar, junto aos estudantes, considerando a programação acadêmica do curso, um fluxo curricular compatível com seus interesses e possibilidades de desempenho acadêmico; orientar a tomada de decisões relativas a matrícula, trancamentos e outros atos de interesse acadêmico, além das outras atribuições previstas nesse regime.

Nesse contexto, para atingir os avanços possíveis na perspectiva de aperfeiçoar o que está sendo desenvolvido no curso e na instituição, há uma necessidade cada vez maior da articulação entre docentes e estudantes do curso sobre as atuais limitações e possibilidades de mudanças para a concretude de uma orientação acadêmica eficaz, qualificada e transformadora. Nesse sentido, o objetivo desta investigação foi avaliar o papel da orientação acadêmica e desafios atuais relacionados a essa prática pedagógica na 
percepção de docentes e discentes do curso de Odontologia da UFRN.

\section{METODOLOGIA}

Trata-se de um estudo descritivo, exploratório e de natureza quantiqualitativa, desenvolvido no Departamento de Odontologia da UFRN. A pesquisa compreendeu a percepção de docentes e discentes sobre a orientação acadêmica e os desafios atuais dessa prática pedagógica durante a formação em Odontologia. Assim, foram convidados a participar do estudo os docentes que já haviam realizado a função de orientação acadêmica e estudantes regularmente matriculados em todos os períodos do curso, com exceção do primeiro.

A seleção desses períodos se deveu ao fato de se objetivar capturar as percepções dos discentes quanto ao processo da orientação acadêmica durante o momento de ingresso na universidade (períodos iniciais), e conclusão do curso (períodos finais). Foi utilizada como técnica de coleta de dados a aplicação de questionários semiestruturados, previamente testados, e aplicados durante o $2^{\circ}$ semestre letivo de 2018. Todos os sujeitos participantes foram previamente informados sobre os propósitos da pesquisa, e só foram incluídos os que aceitaram colaborar, por meio da assinatura do Termo de Consentimento Livre e Esclarecido. Foram excluídos da análise todos os questionários que possuíam erros de preenchimento. O projeto de pesquisa foi aprovado pelo Comitê de Ética em Pesquisa Central da UFRN (CAAE 13202419.6.0000.5292, parecer 3.455.507/2019).

$\mathrm{O}$ instrumento não exigia a identificação do estudante e era composto por questões objetivas e discursivas relativas à orientação acadêmica, dentre elas: a função do orientador, em qual período se deu o primeiro encontro, em quais situações precisou de orientação, quais os momentos que tiveram ou gostariam de receber orientação e soluções para melhorar a orientação acadêmica. Já o questionário dos docentes era composto apenas por questões abertas, relacionadas à sua prática como orientador acadêmico, atividades realizadas, desafios encontrados durante o exercício dessa função e sugestões para superar tais dificuldades.

Para a análise dos dados, as respostas referentes às questões objetivas do questionário aplicado aos estudantes foram analisadas quantitativamente por estatísticas descritivas. As informações referentes às questões discursivas dos questionários dos discentes e docentes foram interpretadas qualitativamente pelo processo de análise de conteúdo ${ }^{11}$.

\section{RESULTADOS E DISCUSSÃO}

Participaram da pesquisa 15 professores efetivos, 46 estudantes dos períodos iniciais $\left(2^{\circ}\right.$ ao $5^{\circ}$ períodos) e 60 dos períodos finais $\left(6^{\circ}\right.$ ao $\left.9^{\circ}\right)$ do curso de Odontologia, totalizando 106 discentes.

As percepções dos estudantes em relação à orientação acadêmica apresentaram diferenças de acordo com o momento da graduação em que se encontravam. Nesse sentido, quando questionados sobre o conhecimento acerca da função do orientador acadêmico, 32 (70\%) dos estudantes dos períodos iniciais sinalizaram que não sabiam, enquanto 38 (63\%) dos que cursavam os períodos finais responderam que sim. As narrativas dos estudantes em linhas gerais corroboram com o que está sinalizado como obrigações do orientador acadêmico dispostas na resolução institucional, como pode-se observar nos relatos dos estudantes.

"Orientar os alunos em relação a qualquer dúvida curricular, disciplinas a serem pagas, dificuldades individuais de cada aluno no decorrer do curso, além de encontrar sempre formas da produtividade do acadêmico ser satisfatória” $\left(2^{\circ}\right.$ período).

"Orientar os alunos na tomada de decisão acadêmica, no que diz respeito a trancar 
disciplinas, quais os prejuízos, quais optativas são mais adequadas aos alunos dependendo do período que estejam $\left(6^{\circ}\right.$ período).

"É o docente responsável por assegurar que o aluno está tendo a vivência acadêmica necessária e esperada. Que está cumprindo com a carga horária esperada, que tem vínculos em projetos e outras ações necessárias para se formar" $\left(8^{\circ}\right.$ período).

Essas atribuições se inserem em uma discussão mais ampla sobre o papel do docente de Odontologia após as mudanças e reestruturações curriculares sofridas a partir das DCN, em que além de se preocupar com a transmissão de conhecimentos, o professor também deve concentrar sua atenção em atividades de acompanhamento do estudante, sob a forma de orientação, de coordenação e integração dos alunos em grupos de pesquisa ${ }^{12,13}$. Para isso, os autores sinalizam que para essa proposta de atuação docente é essencial mudanças nos projetos pedagógicos dos cursos e, sobretudo, nas práticas educativas que orientam a formação em Odontologia, fato que traz à tona resistência de alguns atores envolvidos (docentes e discentes) principalmente daqueles mais arraigados ao ensino tradicional $^{12-14}$.

Quando questionados em relação a conhecer o orientador, apenas 4 (9\%) dos iniciantes e $3(5 \%)$ dos concluintes responderam que não. Entre os estudantes que o conheciam, o momento mais relatado do primeiro encontro se deu no segundo período para os estudantes iniciantes $(23,55 \%)$ e no sexto período para os concluintes $(18,30 \%)$, como ilustrado na figura 1.

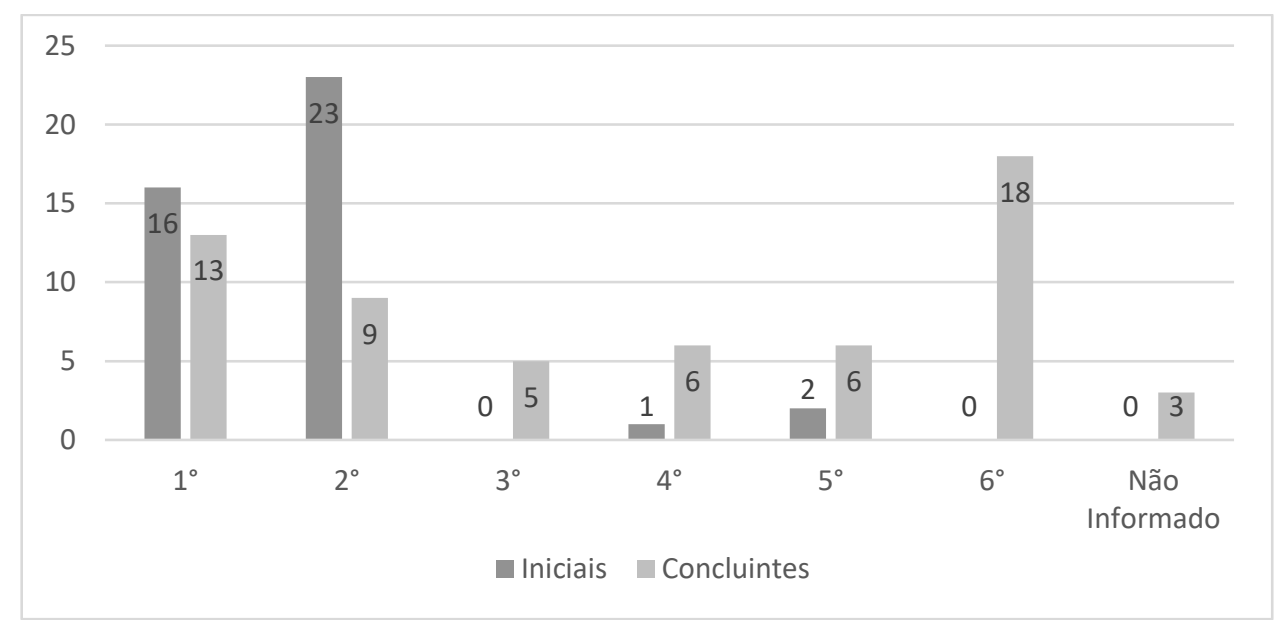

Figura 1. Distribuição das respostas quanto ao período do primeiro encontro com o orientador para estudantes iniciantes $\left(2^{\circ}\right.$ ao $5^{\circ}$ períodos $)$ e concluintes $\left(6^{\circ}\right.$ ao $9^{\circ}$ períodos $)$

O encontro tardio entre estudante-orientador é prejudicial, uma vez que para uma orientação eficaz é importante maior disponibilidade e estabelecimento de vínculo que aproxime os atores do processo, como observado na fala a seguir.
"Todo semestre a minha orientadora se dispõe a me ajudar, pergunta se tem algum problema que precisa ser resolvido, bem como orienta em relação as matérias $e$ disciplinas optativas, isso faz toda a 
diferença, mas eu sei que nem todos os professores aqui são assim" ( $8^{\circ}$ período).

Esta evidência, também observada em outros estudos $^{15,16}$, demonstra a importância da metodologia e da prática pedagógica utilizada pelo corpo docente para facilitar, compreender e aumentar o interesse dos estudantes pela orientação acadêmica e melhorar a interação. Nesse contexto, quando esse encontro não acontece precocemente no início da graduação, quando os estudantes apresentam muitos anseios e dúvidas em relação ao curso, essa relação se torna comprometida.

Somado a isso, a maioria dos encontros entre estudante e orientador ocorreram em sala de aula, quando coincidia o componente curricular em que a turma estava cursando ser ministrado pelo orientador, principalmente nos períodos iniciais. Além disso, 34 (74\%) dos estudantes dos períodos iniciais e 33 (55\%) dos períodos finais ainda não haviam recebido nenhum tipo de orientação acadêmica durante a graduação. Para a parcela que havia recebido orientação, a maioria dos encontros aconteceu apenas uma ou duas vezes, até o momento da pesquisa. Vale destacar que 31 (67\%) dos matriculados nos períodos iniciais e $36(60 \%)$ dos que cursavam a segunda metade do curso afirmaram que em algum momento da trajetória acadêmica precisaram de ajuda e orientação e deles apenas $10(32 \%)$ e $16(46 \%)$ tiverem seu pedido atendido pelo orientador, respectivamente.

Do material textual gerado a partir das perguntas subjetivas quanto às situações em que os estudantes gostariam de receber ou ter recebido orientação acadêmica, as principais situações abordadas foram em relação a horas complementares, matrículas, disciplinas optativas, congressos, cursos voltados para a área, orientações sobre pós-graduação, currículo Lattes, projetos de pesquisa e extensão, estágios e custos relacionados às disciplinas clínicas. As narrativas a seguir ilustram essas observações.

"Gostaria de ter recebido em cada etapa da jornada acadêmica: disciplinas optativas, como ingressar em um projeto, quais horas complementares são necessárias para conclusão do curso, indicação de cursos e disciplinas optativas" $\left(4^{\circ}\right.$ período $)$.

"Orientações relacionadas ao currículo lattes e outras atividades relacionadas à área da pesquisa e pós-graduação" ( $9^{\circ}$ período).

"No início das clínicas, em relação à compra de material, fiquei meio perdido. Na elaboração de trabalhos, resumos para congressos e no maior estímulo para continuar no curso, tirando dúvidas sobre mercado de trabalho" ( $5^{\circ}$ período).

Nesse cenário, fica evidente a necessidade de incorporar os princípios das DCN, que sinalizam o comportamento proativo dos estudantes em relação ao processo de ensino-aprendizagem e o papel do professor como facilitador ${ }^{16,17}$. Dessa forma, com a utilização de uma metodologia de ensino contemporânea, os professores deverão ser capazes de refletir sobre sua importância na condução da orientação acadêmica como facilitadores, contribuindo para a integração dos estudantes à vida universitária, colocando-os no papel de protagonistas de seus aprendizados quanto às atividades acadêmicas $6,17,18$,

Outro achado importante nesse contexto foi que em diversas vezes houve falta de conhecimento acerca das atribuições do orientador acadêmico por parte do discente, o que aconteceu quando os mesmos indicaram situações não condizentes com as atividades de orientação acadêmica.

$\mathrm{Na}$ perspectiva de qualificar esse processo, os estudantes sinalizaram algumas sugestões para melhorar a atuação dos orientadores acadêmicos no âmbito do Departamento de Odontologia. Dentre elas, a precocidade necessária do primeiro encontro, maior comunicação entre estudante e orientador, organização acadêmica entre as 
atividades em um mesmo semestre, frequência dos encontros, acompanhamento das atividades acadêmicas e horas complementares, entre outras. Nesse sentido, as falas a seguir destacam algumas dessas sugestões.

"Conversa presencial desde o primeiro período do curso para se apresentar e falar sua função, estou prestes a me formar $e$ nem conheço meu orientador acadêmico ainda" ( $9^{\circ}$ período).

"Criar um canal de comunicação melhor entre orientador e aluno, mesmo online. Oficializar as reuniões no mínimo semestral para discutir temas pertinentes à turma e ao semestre" ( $7^{\circ}$ período).

"Realizar encontros presenciais obrigatórios para avaliar os desempenhos dos alunos. Principalmente em relação às horas obrigatórias, pois fica tudo no final do curso, e ficamos ainda mais cheios, junto com o TCC" (9 $9^{\circ}$ período).

"Orientação quanto à indicação de auxílios que a universidade dispõe desde o primeiro período" ( $2^{\circ}$ período).

Diante desse panorama, as sugestões dos estudantes sinalizam que os orientadores acadêmicos se tornem mais presentes no cotidiano universitário desde o primeiro período, com orientações precoces sobre as suas obrigações e mediando o processo de formação acadêmica em todas as fases do curso.

Já em relação às percepções dos docentes, os 15 professores participantes desta pesquisa atuavam na formação acadêmica nas áreas de concentração de Odontologia Preventiva e Social, Dentística, Ortodontia, Endodontia, Periodontia, Implantodontia, Odontopediatria e Prótese Dentária. No tocante ao período que exerceram a função de orientadores acadêmicos, foi observado um intervalo médio de 2 a 4,5 anos.

No entanto, a maioria dos docentes relatou não ter recebido nenhuma orientação ou capacitação para atuar como orientador acadêmico, salvo algumas exceções, marcadamente entre professores novatos no curso. Para eles, os principais motivos sinalizados para terem sido escolhidos para a atividade de orientação estavam relacionados ao ingresso na instituição como docente efetivo, experiência e/ ou o envolvimento com questões burocráticas e acadêmicas do curso, como observado nas seguintes narrativas.

"Tinha acabado de passar no concurso para docente na [item ocultado], e então a coordenadora do curso na época me convidou para ser orientadora acadêmica";

"Fui escolhido pois eu era novo na instituição, fazia parte do colegiado de curso e me envolvia com as questões acadêmicas do curso";

"Não sei quais os critérios à época. Talvez o meu envolvimento e compromisso com o curso de graduação, talvez minha experiência acadêmica".

Dentre as principais atividades citadas pelos orientadores acadêmicos no exercício de suas funções estão, em ordem decrescente, a orientação e validação de matrículas, orientação em relação às atividades complementares, acompanhamento da integralização dos componentes curriculares, tirar dúvidas acerca do curso, reuniões semestrais com a turma, acompanhamento de situações pontuais, como em casos de intercâmbios acadêmicos, quando os estudantes retornam e precisam se inserir novamente nas atividades curriculares regulares.

No que tange aos principais desafios ou dificuldades encontradas no exercício da função de orientador acadêmico, os docentes destacaram a falta de clareza sobre como deve atuar um orientador acadêmico, a falta de conhecimento da estrutura curricular do curso, a relação entre as disciplinas (co-requisito, pré-requisito, etc), o encontro com a turma nos primeiros semestres, 
pois os estudantes se encontram no ciclo básico e a maioria dos orientadores atuam no ciclo profissionalizante. Alguns autores sinalizam que essas dificuldades assumem particular relevância nos cursos de graduação em saúde, onde os diferentes cenários de ensino-aprendizagem, as inúmeras atribuições dos docentes, a sobrecarga de trabalho e a complexidade da estrutura curricular tornam o desafio de mudanças ainda maior ${ }^{19,20}$. Nesse sentido, os professores elencaram algumas sugestões com propostas para superar tais desafios, apresentadas nas falas a seguir.

"Que seja elencada uma lista de obrigações do orientador acadêmico bem como do aluno";

"Capacitação dos futuros orientadores antes do início das atividades e reuniões periódicas de acompanhamento, sob a responsabilidade da coordenação do curso";

"Realização de oficinas, cursos e discussões para que possamos estar mais cientes do nosso papel, bem como saber orientar melhor os alunos em questões pertinentes";

"Realização a cada início de semestre de encontros com os alunos para falar sobre a orientação acadêmica no semestre";

"Implementação de um manual de orientação acadêmica".

$\mathrm{Na}$ Odontologia, a qualidade do ensino está relacionada, entre tantos fatores, com a qualificação e educação permanente (tanto técnica quanto didático-pedagógica) dos professores $^{21}$. Dessa forma, considerando os resultados é possível refletir que apesar de toda potencialidade da orientação acadêmica como uma estratégia pedagógica na formação, ela ainda é subaproveitada tanto pelos estudantes quanto pelos professores na UFRN.

Essa fragilidade apresentada nos relatos de experiências dos professores e estudantes evidencia que a educação permanente constitui um espaço privilegiado de reflexão sobre a prática docente, sobretudo voltada para o papel do professor orientador acadêmico. Nesse sentido, o papel da educação permanente seria estimular, em todos os cenários, a problematização das vivências, e ampliar a troca de experiências entre os participantes, favorecendo o desenvolvimento pessoal e do processo de trabalho ${ }^{21,22}$. Em um universo de possibilidades, as propostas de desenvolvimento docente em Odontologia que vêm sendo implementadas e sinalizadas nos cursos de graduação, tem levado à reflexão sobre as mais diversas práticas docentes em âmbito nacional e precisam ser divulgadas.

É notório que as propostas de mudanças nas práticas pedagógicas que impactam diretamente na qualidade do ensino e formação em Odontologia deva prever elementos nos quais fique clara a compreensão das DCN, em sua essência e princípios, sobretudo, em relação ao papel do docente e dos estudantes no processo de ensinoaprendizagem ${ }^{1,6,23}$. Dessa forma, torna-se essencial o comprometimento docente, e que a instituição assuma o seu papel na capacitação e qualificação dos professores e da valorização da função da orientação acadêmica neste processo, com vistas aos resultados almejados, tanto por parte dos estudantes quanto dos professores.

Diante da complexidade que envolve as mudanças de práticas pedagógicas no ensino em saúde, os resultados dessa investigação foram essenciais para nortear o NDE e a coordenação do curso de Odontologia da UFRN na superação das dificuldades e fragilidades encontradas por estudantes e professores, em relação à orientação acadêmica no âmbito da formação em Odontologia.

Dentre as estratégias que já foram encaminhadas pela instituição, destaca-se a criação de um Manual de Orientação Acadêmica para o curso de Odontologia, com informações acerca da 
atuação, atribuições e metodologias que deverão ser utilizadas na prática pedagógica de orientação acadêmica, nos próximos anos, no curso de Odontologia da UFRN. Somado a isso, também estão sendo planejadas oficinas estratégicas de capitação docente, diante a necessidade de investir na educação permanente e formação de professores, para atuar como facilitadores do processo de orientação acadêmica no ensino em Odontologia. Por fim, espera-se que os resultados discutidos nessa investigação sirvam também de reflexão sobre essa prática pedagógica em outras IES brasileiras.

\section{CONCLUSÃO}

Mesmo diante sua potencialidade como uma estratégia pedagógica na integração dos estudantes à vida universitária, a orientação acadêmica ainda é subaproveitada por discentes e docentes.

\section{ABSTRACT \\ Academic tuition: perceptions of students and professors concerning pedagogical practice in dental education}

This study aimed to evaluate the role of academic tuition and the challenges related to this pedagogical practice during academic education in Dentistry, considering the perception of professors and students. This is a quantitativequalitative study, developed in the Dental school of Federal University of Rio Grande do Norte (UFRN). Data was collected by means of semistructured questionnaires, answered by 106 students, and 15 professors with expertise in academic tuition. Data analysis was descriptive and a qualitative analysis was carried out using the content analysis method. Student perceptions showed weaknesses in the performance of academic tuition, mostly concerning the relationship between tutor and students, frequency of discussions, as well as the method adopted. The professors pointed out the need for permanent education strategies, aiming at their qualifications to the tuition. It is concluded that there is an underuse of this pedagogical practice in dentistry education. To overcome this weakness and the challenges inherent to the practice, in the view of the students, it is necessary that the tutors become more present during the onset of their university life by early directions on their responsibilities and supervision of the education process along the graduation. For professors, it is necessary to provide permanent strategies for academic tuition improvements.

Descriptors: Education, Dental. Faculty, Dental. Staff Development.

\section{REFERENCIAS}

1. Fonseca EP. As diretrizes curriculares nacionais e a formação do cirurgião-dentista brasileiro. J Manag Prim Health Care. 2012; 3(2):158-78.

2. Warmling, CM, Marzola, NR, Botazzo C. Da autonomia da boca: práticas curriculares e identidade profissional na emergência do ensino brasileiro da odontologia. Hist Ciênc Saúde Manguinhos. 2012; 19 (1):181-95.

3. Zilbovicius C, Araujo ME, Botazzo C, Frias AC, Junqueira SR, Junqueira CR. A paradigm shift in predoctoral dental curricula in Brazil: evaluating the process of change. J Dent Educ. 2011,75 (4):557-64.

4. Freitas SFT, Calvo MCM, Lacerda JT. Saúde coletiva e novas diretrizes curriculares em odontologia: uma proposta para graduação. Trab Educ Saúde. 2012; 10(2):223-34.

5. Junges R, Stello RS, Rösing CK, Samuel SMW. Impact of new curriculum implementation on student experience in a school of dentistry in Brazil. Braz Oral Res. 2011; 25(6):478-84.

6. Noro LRA, Farias-Santos BCS, Sette-deSouza PH, Pinheiro IAG, Borges REA, Nunes LMF, et al. O professor (ainda) no centro do processo ensino-aprendizagem em Odontologia. Rev ABENO. 2015; 15(1): 211.

7. Secco LG, Pereira MLT. Concepções de qualidade de ensino dos coordenadores de graduação: uma análise dos cursos de 
odontologia do Estado de São Paulo. Interface Comun Saúde Educ. 2010;8(15) :313-30.

8. Scorzoni MF, Bueno SMV, Coscrato G. O currículo e as implicações dos novos paradigmas educacionais na formação do enfermeiro. Saúde Transform Soc. 2013; 4(1):11-5.

9. Ferreira NP, Dantas TS, Sena-Filho M, Rocha DG. Clínica integrada e mudança curricular: desempenho clínico na perspectiva da integralidade. Pesqui Bras Odontop Clín Integr. 2012;12(1):33-9.

10. Ministério da Educação. RESOLUÇÃO No 171/2013-CONSEPE, de 5 de novembro de 2013. Regulamento dos Cursos Regulares de Graduação da Universidade Federal do Rio Grande do Norte. Natal, RN. 2013. [Acesso em: 01/03/2019]. Disponível em: https://ufrn.br/resources/documentos/regula mentos/regulamento-dos-cursos-regularesde-graduao-da-UFRN.pdf.

11. Bardin, L. Análise de conteúdo.70 ed. São Paulo: Almedina; 2011.

12. Toassi RFC, Souza JM, Baumgarten A, Rösing CK. Avaliação curricular na educação superior em odontologia: discutindo as mudanças curriculares na formação em saúde no Brasil. Rev ABENO. 2012; 12(2):170-7.

13. Toassi RFC. Integrated curriculum for teaching dentistry: new directions for training in the field of healthcare. Interface (Botucatu). 2012;16(41):529-42.

14. Reul MA, Lima ED, Irineu KN, Cardoso RSC, Costa EMMB, Madruga RCR. Metodologias ativas na graduação em Odontologia e a contribuição da monitoria. Rev ABENO. 2016; 16 (2): 62-8.

15. Júnior AMLF, Miranda NR, Assunção R, Silva SA, Oliveira FAM, Oliveira RG. Percepção de estudantes de Odontologia sobre metodologias ativas no processo de ensino-aprendizagem. Rev ABENO. 2016; 16(3):66-77.

16. Semim GM, Souza MCBM, Corrêa AK. Professor como facilitador do processo ensino-aprendizagem: visão de estudante de enfermagem. Rev Gaúcha Enferm. Porto Alegre (RS). 2009;30(3):484-91.

17. Noro LRA, Farias-Santos BCS, Sette-deSouza PH, Pinheiro IAG, Borges REA, Cruz RKS, et al. Revisiting the Basic Cycle: Prospects by Undergraduates' Students of a Brazilian Dentistry School. Pesqui Bras Odontop Clin Integr. 2019; 19(1):e3901.

18. Lage RH, Almeida SKTT, Vasconcelos GAN, Assaf AV, Robles FRP. Ensino e Aprendizagem em Odontologia: Análise de Sujeitos e Prática. Rev Bras Edu Med. 2017; 41 (1): 22-9.

19. Borges MC, Miranda CH, Santana RC, Bollela VR. Avaliação Formativa e aprendizado na saúde. Medicina (Ribeirão Preto). 2014;47(3):324-31.

20. Deus JM, Alves DRN, Figueiredo RR, Silva MMM, Amaral AF, Bollelal VR. Aula centrada no aluno versus aula centrada no professor: Desafios para a mudança. Rev Bras Educ Med. 2014, 38(4):419-26.

21. Borges REA, Noro LR. Coerência entre editais de concurso público para professores e Diretrizes Curriculares dos cursos de Odontologia. Rev ABENO. 2016; 16(4):2939.

22. Lazarin CA, Francischetti I. Educação Permanente: uma ferramenta para o desenvolvimento docente na graduação. Rev Bras Educ Méd. 2010;34(4):481-6.

23. Brehmer LCF, Ramos FRS. Experiências de integração ensino-serviço no processo de formação profissional em saúde: revisão integrativa. Rev Eletr Enferm. 2014; 16(1):228-37.

\section{Correspondência para:}

Raul Elton Araújo Borges

e-mail: raullelton@hotmail.com

Av. Sen. Salgado Filho, 1787 - Lagoa Nova, 59056-000 Natal/RN 